\title{
Multiple solutions and transient chaos in a nonlinear flexible coupling model
}

\author{
Jerzy Margielewicz ${ }^{1}$ - Damian Gąska ${ }^{1}$ (1) · Tadeusz Opasiak ${ }^{1} \cdot$ Grzegorz Litak $^{2}$
}

Received: 29 March 2021 / Accepted: 13 September 2021 / Published online: 30 September 2021

(c) The Author(s) 2021

\begin{abstract}
This paper investigates the nonlinear dynamics of a flexible tyre coupling via computer modelling and simulation. The research mainly focused on identifying basins of attraction of coexisting solutions of the formulated phenomenological coupling model. On the basis of the derived mathematical model, and by assuming ranges of variability of the control parameters, the areas in which chaotic clutch movement takes place are determined. To identify multiple solutions, a new diagram of solutions (DS) was used, illustrating the number of coexisting solutions and their periodicity. The DS diagram was drawn based on the fixed points of the Poincaré section. To verify the proposed method of identifying periodic solutions, the graphic image of the DS was compared to the three-dimensional distribution of the largest Lyapunov exponent and the bifurcation diagram. For selected values of the control parameter $\omega$, coexisting periodic solutions were identified, and basins of attraction were plotted. Basins of attraction were determined in relation to examples of coexistence of periodic solutions and transient chaos. Areas of initial conditions that correspond to the phenomenon of unstable chaos are mixed with the conditions of a stable periodic solution, to which the transient chaos is attracted. In the graphic images of the basins of attraction, the areas corresponding to the transient and periodic chaos are blurred.
\end{abstract}

Keywords Nonlinear vibrations $\cdot$ Chaos $\cdot$ Transient chaos $\cdot$ Diagram of solutions $\cdot$ Basins of attraction

\section{Introduction}

In flexible couplings, the nonlinearity of the system is mainly due to the mechanical characteristics of the flexible connector. For example, the connectors of tyre couplings are usually made of materials such as neoprene, hytrel and urethane (among others), in which steel spring inserts are moulded, and suitably shaped depending on the design of the coupling [1-3]. Mechanical characteristics of the clutch play a key role during model tests, because a small change in the application of the external load can lead to different solutions. While the results of simulations based on linear

Technical Editor: Pedro Manuel Calas Lopes Pacheco.

Damian Gąska

damian.gaska@polsl.pl

1 Faculty of Transport and Aviation Engineering, Silesian University of Technology, Krasińskiego 8, 40-019 Katowice, Poland

2 Faculty of Mechanical Engineering, Lublin University of Technology, Nadbystrzycka 36, 20-618 Lublin, Poland models are predictable, robust against small parametric and initial conditions changes, and easily interpretable, the same cannot be said about nonlinear models. Solutions of nonlinear models are sensitive to system parameter and initial conditions. Additionally, non-periodic (chaotic) solutions are present [4]. Consequently, small perturbations coming from the coupling to the ambient conditions including the noise in the system excitation, can drive the system to bifurcations and/or solutions unpredictability. The study of chaotic phenomena has become widespread since the seminal publication of Edward Lorenz in 1963, where he presented the results of computer simulations of a simplified model of fluid convection [5]. That work demonstrated that small changes in the initial conditions in a small area of the atmosphere can cause major changes in other areas of the atmosphere. The appearance of this publication led to the development of a new scientific discipline known as chaos theory.

Chaos theory has been applied in different areas, among others, in mechanical systems [6,7], vehicle suspensions $[8,9]$, electrical circuits [10], robotics [11], medicine [12] or economics [13]. In these works, it was shown that the 
chaotic behaviour of the dynamical system takes place when the system is sensitive to a change in the initial conditions. In particular, many numerical tools can be used to identify chaotic motion, for example, estimating the largest Lyapunov exponent $[14,15]$, plotting a bifurcation diagram (also known as the Feigenbaum steady-state diagram [16, 17]), plotting a Poincaré cross section $[18,19]$ and analysing the Fourier spectrum. An important numerical tool, useful in the study of nonlinear systems, are short-time Fourier transform (STFT) [20] and wavelet transform [21, 22]. Through them, it is possible to evaluate the evolution of harmonic components in the amplitude-frequency spectrum over time. An alternative way to identify chaotic motion zones is to run the $0-1$ test [23] or delineation of the correlation dimension diagram [2].

An important aspect of nonlinear dynamics research is the identification of coexisting multiple (subharmonic) solutions for different initial conditions, which in consequence boils down to seeking attractors. In general terms, this issue studies time series defining trajectories aimed at specific attractors. In contrast to the procedure for estimating the largest Lyapunov exponent, the entire set of initial conditions located at different points of the phase plane are considered. The results of these studies are most often presented in the form of multicoloured maps showing the structure of the basins of attraction, which were plotted with reference to the Duffing equation [24], magnetic pendulum [6] and other dynamic systems [25].

During the study of nonlinear dynamic systems, you can encounter the so-called phenomenon of transient chaos. This phenomenon was observed in electric circuits [26], standard nonlinear dynamical models such as the logistic mapping [27], Duffing equation [28] and during experimental tests of the pendulum model [29]. In general, the term "transient chaos" is used when describing a chaotic response, which after some time becomes a periodic or quasi-periodic response [30,31]. Intervals of transient chaos are observed in systems with two or more solutions characterized by basins of attraction with a fractal border (the so-called Mielnikow's chaos [32-34]). In nonlinear dynamical systems, the phenomenon of crisis is considered to be the source of the most sudden changes in chaotic dynamics. In the work [35], it has been shown that the phenomenon of crisis occurs as a result of the collision of a chaotic attractor with a coexisting fixed point or periodic orbit. For this reason, the phenomena of transient chaos and crisis are interrelated. Research of transient chaos phenomenon in real machine drive systems appears to be very important, because it often occurs in computer simulations of very simple theoretical systems.

The subject of the research included in this work is the assessment of possible solutions of the nonlinear flexible coupling model under given load conditions. Numerical experiments were carried out based on the characteristics, described in the laboratory experiment, describing causal relations between the torque moment and the angular displacement. The results of computer simulations are presented in the form of multicoloured maps of the largest Lyapunov exponent, Poincaré cross sections, bifurcation diagrams, basins of attraction and diagrams indicating the number of coexisting solutions and their periodicity (DS). Based on them, one can determine the conditions for the occurrence of the so-called phenomena of transient chaos. Numerical algorithms evaluating the short-term Fourier transformation (STFT) were used for its quantitative and qualitative description [20]. Another approach is the use of the wavelet transform, which in relation to STFT is characterized by a much better resolution in the low frequency range [21]. These transformations are most often used in technical diagnostics to monitor the technical condition of machines. Nevertheless, they are also used in model studies, for graphical visualization of the evolution of harmonic components over time. Regardless of the applied method of transforming the signal from the time domain to the frequency domain, it is possible to determine at what moment the chaotic solution turns into a periodic solution.

\section{Formulation of the mathematical model}

The object of the model tests is the flexible tyre coupling, whose mechanical characteristics were identified based on laboratory tests, and preliminary results were published in [2]. The measurement data recorded in laboratory conditions are very well approximated by the third-degree polynomial function, which is presented graphically in the diagram (Fig. 1b). Laboratory tests were carried out in a system of two induction motors connected by a flexible coupling (Fig. 1a). One of the motors was operated in steady state, which ensured constant angular velocity of the rotor. In contrast, the angular velocity of the second motor was controlled via an inverter. The external load acting on the coupling, induced by the change of the rotational speed of the resistance motor, is the source of the changing torque. The difference in angular displacements recorded on both clutch plates was measured through a stroboscopic speed measurement disc. Within the range of small angular displacements up to $0.07 \mathrm{rad}$ (low load values), the coupling works as a linear mechanical system. Nevertheless, in the case of large angular displacements or system overloads, it is necessary to consider the entire nonlinear characteristic of the flexible coupling (Fig. 1b) [2].

The phenomenological model investigated here involves two rotating discs with moments of inertia $J_{1}$ and $J_{2}$, which are coupled by a nonlinear spring element $c$ and a linear viscous damper $b$ (Fig. 2). The movement of the system is generated by an external harmonic torque $M_{N}$, which is 
(a)

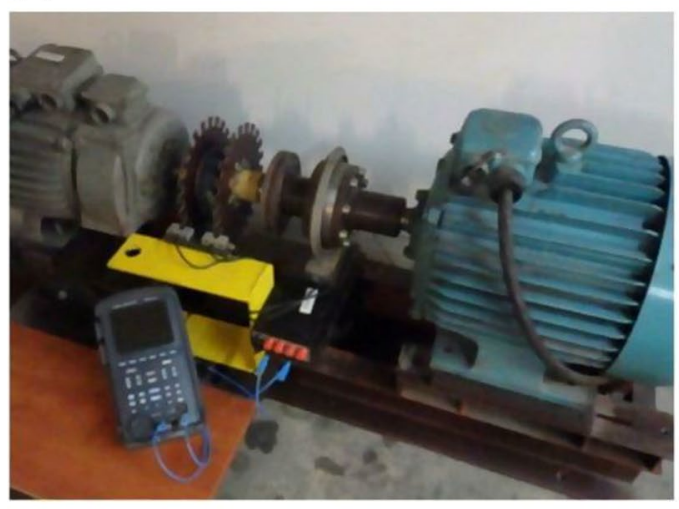

(b)

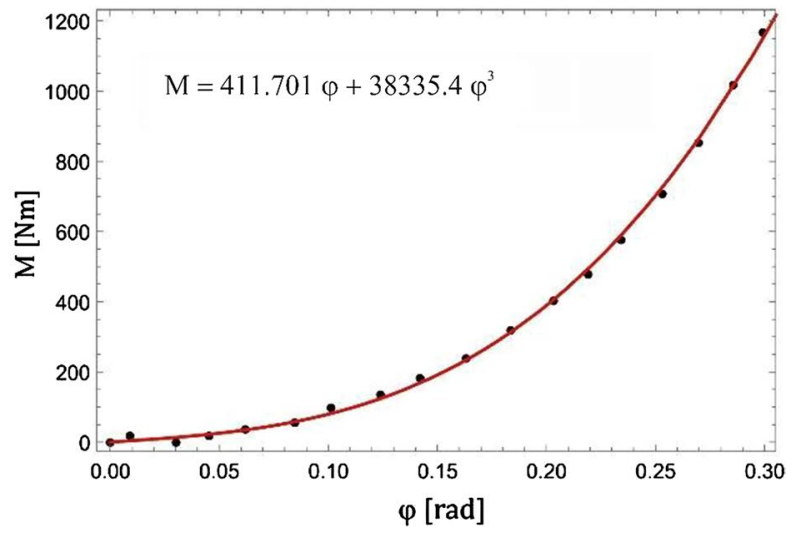

Fig. 1 Experimental tests: a construction of the laboratory stand, b mechanical characteristics identified [2]

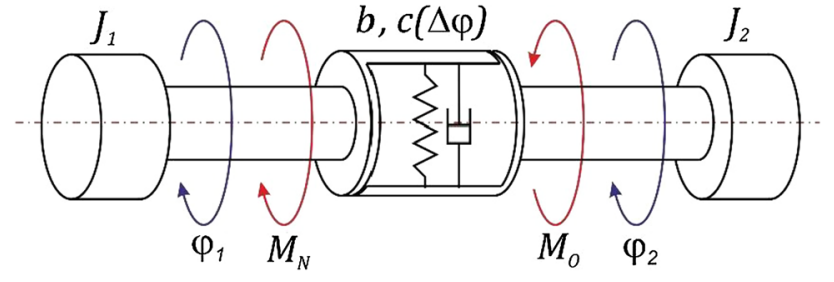

Fig. 2 A phenomenological model of a flexible tyre coupling

applied to the active disc $J_{1}$. The passive disc $J_{2}$ is affected by a constant load moment $M_{O}$.

From a theoretical point of view, the differential equations of motion of mechanical systems can be derived by various methods, both classical and non-classical [2]. Regardless of which method is used, the following mathematical model was derived:

$$
\begin{aligned}
& J_{\text {red }}=\frac{J_{1} J_{2}}{J_{1}+J_{2}}, \alpha=\frac{c_{1}}{c_{0}}, \delta=\frac{b}{J_{\text {red }} \omega_{0}}, \omega_{0}^{2}=\frac{c_{0}}{J_{\text {red }}}, \omega=\frac{\omega_{W}}{\omega_{0}}, \\
& \tau=\omega_{0} t, p_{0}=\frac{M_{O}}{\omega_{0}^{2} J_{2}}, p_{1}=\frac{M_{N}}{\omega_{0}^{2} J_{1}}, \mu=\frac{p_{0}}{p_{1}} .
\end{aligned}
$$

The structure of the mathematical model of a flexible tyre clutch is similar to the Duffing equation. At the same time, five parameters have an influence on its dynamics. Differential Eq. (2) provides a formal basis for quantitative and qualitative model research, which is the objective of this manuscript.

\section{Results of model tests}

Model tests were carried out for a flexible tyre coupling, the basic purpose of which is to transfer large loads with relatively small dimensions and a compact structure. The motion of the tested system is induced by a four-pole induction motor with a rated power $P=7.5 \mathrm{~kW}$ and rotational

$\left\{\begin{array}{l}J_{1} \ddot{\varphi}_{1}+b\left(\dot{\varphi}_{1}-\dot{\varphi}_{2}\right)+c_{0}\left(\varphi_{1}-\varphi_{2}\right)+c_{1}\left(\varphi_{1}-\varphi_{2}\right)^{3}=M_{N} \cos \left(\omega_{W} t\right) \\ J_{2} \ddot{\varphi}_{2}-b\left(\dot{\varphi}_{1}-\dot{\varphi}_{2}\right)-c_{0}\left(\varphi_{1}-\varphi_{2}\right)-c_{1}\left(\varphi_{1}-\varphi_{2}\right)^{3}=-M_{O}\end{array}\right.$

where $c_{0}$ and $c_{l}$ represent the static characteristic coefficients of the coupling.

In the following equations, a new variable was introduced $u=\varphi_{1}-\varphi_{2}$ defined as the difference in angular displacements of the flexible tyre coupling discs. As a result of such substitution, the system of Eqs. (1) is reduced to one differential equation whose dimensionless form is given by:

$\ddot{u}+\delta \dot{u}+u+\alpha u^{3}=p_{1}(\mu+\cos (\omega \tau))$,

where: speed $n_{S}=1450 \mathrm{~min}^{-1}$. During the computer simulations, ideally rigid shafts connecting the clutch with the electric motor and the remaining components of the drive system were used. The adoption of such model assumptions was aimed at eliminating the dynamic influences caused by its operation. They are caused, among other things, by elastic deformations.

As a result of such assumptions, the influence of parameters characterizing the mechanical properties of the clutch on its dynamics can be assessed. In addition, while carrying out numerical experiments, the value of the load torque 
Table 1 Parameters characterizing the tested coupling

\begin{tabular}{lll}
\hline Name & Symbol & Value \\
\hline Nominal torque & $M_{n}$ & $1100 \mathrm{Nm}$ \\
Maximal torque & $M_{\text {MAX }}$ & $3250 \mathrm{Nm}$ \\
The diameter of the flexible connector & $d$ & $316 \mathrm{~mm}$ \\
The weight of the clutch & $Q$ & $520 \mathrm{~N}$ \\
Mass moment of inertia & $J$ & $0.46 \mathrm{kgm}^{2}$ \\
Static characteristics of the coupling & $c_{0}$ & $411.7 \mathrm{Nm}^{-1}$ \\
Static characteristics of the coupling & $c_{1}$ & $38,335.4 \mathrm{Nm}^{-1}$ \\
\hline
\end{tabular}

$M_{O}$, loading the coupling at the level of $80 \%$ of the torque value $M_{N}$ was assumed. The equivalent viscous damping coefficient $\delta$ captures the total energy losses in the flexible coupling clutch and resistance to motion in the drive system bearings. Numerical values, taking into account catalogue data and identified during laboratory tests, are presented in Table 1.

In the first stage of model tests, areas where clutch movement is chaotic were identified. All the results of computer simulations included in this chapter were obtained based on dimensionless parameters characterizing the mathematical model of a tyre clutch: $\delta=0.06, \alpha=93.114, \mu=0.8, p_{1}=1$. The results of computer simulations determined in the course of operation were presented in relation to the steady state. It was assumed a priori that such a move occurs after 500 periods of external stress. On the other hand, the fixed points of the Poincaré cross section and the phase trajectories were identified in a time window with a width of 20 periods of excitation.

\subsection{Identification of chaotic motion zones}

To identify of chaotic motion zones, the largest Lyapunov exponent was calculated, which was depicted in the form of a three-dimensional multicoloured map. This indicator is one of the key concepts of chaos theory, through which it is possible to distinguish unpredictable chaotic behaviour. In our research, the largest Lyapunov exponent was estimated based on the given equation:

$\lambda=\lim _{\varepsilon(0) \rightarrow 0, n \rightarrow \infty} \frac{1}{n \tau} \sum_{i=1}^{n} \ln \left(\frac{\varepsilon_{i}(\tau)}{\varepsilon(0)}\right)$

In this equation, $\varepsilon_{i}(\tau)$ represents a vector containing, at the same moment of time, the trajectory of the studied system with the reference trajectory. At the moment $\tau=0$, both trajectories are located in close proximity, and the distance between them in the phase plane is defined by the parameter $\varepsilon(0)=10^{-5}$. In practical applications, the Lyapunov exponent estimation method is performed by averaging over many iterations, in an adequate immersion space. In the Eq. (3), $n$ corresponds to the number of averaged phase trajectories. The phase space that we adopted when estimating the largest Lyapunov exponent is determined by displacement and velocity $(u, \dot{u})$. To obtain satisfactory resolution, it is advisable to carry out many computer simulations, which significantly extends the time cost of numerical calculations. Acceptable resolution was achieved by dividing the variation ranges of the control parameters $\omega$ and $p$ defining the abscissa and ordinate axes into 500 intervals, which consequently gives 250,000 computer simulations to generate a single three-dimensional diagram of the largest Lyapunov exponent.

The chaotic movement occurs when the value of the largest Lyapunov exponent $\lambda$ is located above the transparent plane in green (Fig. 3). Otherwise, the trajectories are aimed at stable points or periodic orbits. The chart shows that the areas of unpredictable clutch operation $\lambda>0$ are arranged in bands, which can be approximated with a third-degree polynomial function with high accuracy. It is also worth noting that as the value of the control parameter $p_{l}$ increases, the width of the chaotic motion zones increases. With respect to the given cross sections of the three-dimensional map of the largest Lyapunov exponent $p_{l}=1$ (Fig. 4), DS diagram was created to form a formal basis for the identification of multiple periodic solutions.

The basic purpose of the DS diagram is to recognize and search for stable periodic solutions. In general terms, the method is used to identify the so-called fixed points of phase streams, whose origins are located at various points of the studied area of the phase plane. The best results are achieved when considering many initial conditions. However, this approach significantly increases the time for numerical calculations. The current research indicates that it is satisfactory to consider the initial conditions, which are located on

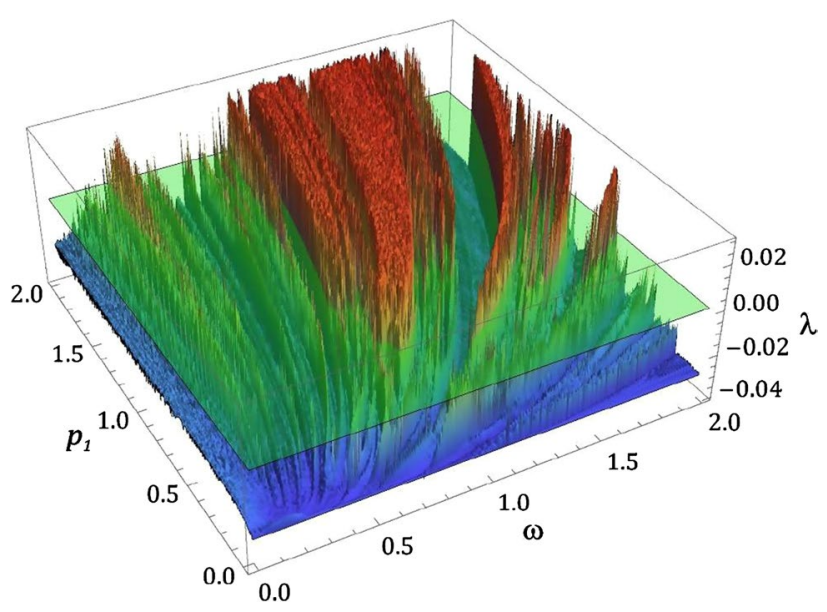

Fig. 3 Three-dimensional map of the largest Lyapunov exponent for determined initial conditions $(u(0)=0, \dot{u}(0)=0)$. Positive values $\lambda$ correspond to chaotic solutions 


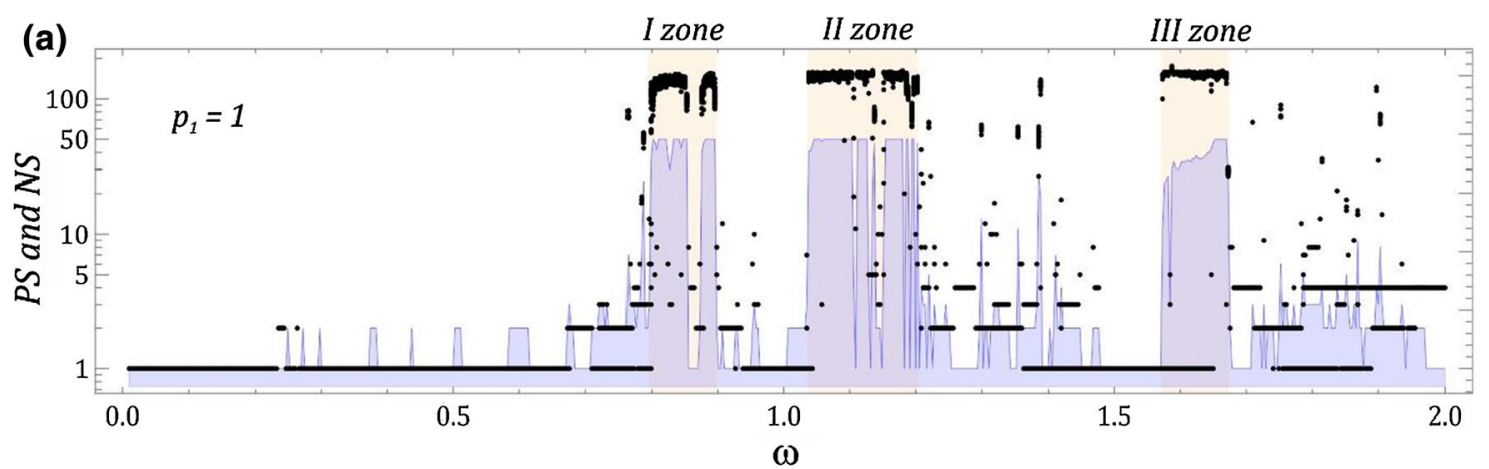

(b)

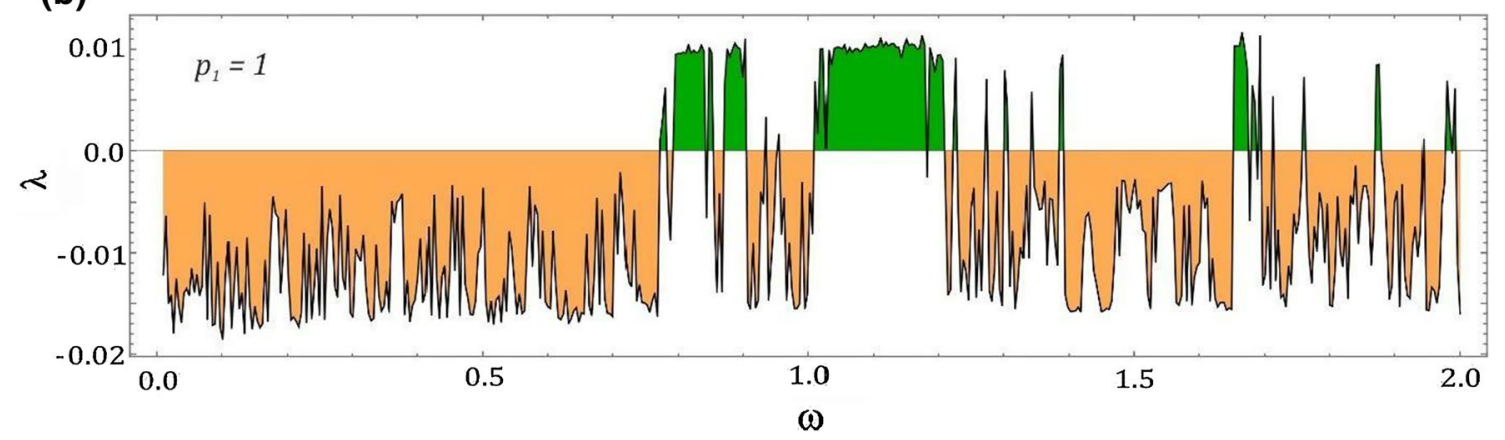

Fig. 4 Diagrams: a DS-periodicity and number of solutions, b distribution of the largest Lyapunov exponent assuming initial conditions $(u(0)=0, \dot{u}(0)=0)$

the main diagonal of the phase plane. This reduces computation time. This approach is justified because the phase stream cyclically crosses the along the main diagonal. The phase coordinates that define the location of fixed points $(x, \dot{x})$, are identified based on the Poincaré cross section. In the case of predictable solutions, the number of fixed points is invariable and independent of the time of the test and directly correlated with the periodicity of the solution, because in the case of periodic answers, the fixed points do not change after iteration from $\tau=n 2 \pi$ to $\tau=(n+1) 2 \pi$.

The proposed numerical tool fulfils its task in relation to chaotic solutions, because in their case the number of identified fixed points is theoretically infinite, but in fact limited by the length of time sequences defining the phase stream. In the DPS diagram, chaotic responses are recognized based on large values representing the periodicity of the solution. In the following, coexisting solutions have been identified that correspond to the given conditions of excitation.

As mentioned before, the solution diagram forms two charts: one is corresponding to the number of coexisting solutions DNS and is represented in the form of a shaded area in blue, whereas the second DPS presented in the form of black points informs about the periodicity of the solution. The most valuable information about nonlinear system dynamics is obtained as a result of a direct comparison of the DNS and DPS diagram. Therefore, it is expedient to present them together on the DS diagram (Fig. 4). The control parameter variability range was divided into 700 equally spaced sections in the plot. Appropriate computer simulations were carried out in relation to low vibration amplitudes of mechanical devices from which energy is harvested.

The most information about the dynamics of a nonlinear system is obtained as a direct result of a comparison between the number of coexisting solutions $N S$ and their periodicity $P S$. For this reason, they are presented in one graph (Fig. 4a). The diagram of solutions DS indicates the existence of single predictable $1 \mathrm{~T}$-periodic solutions in the interval $\omega \in\langle 0,0.67\rangle$. Such solutions also occur between the unpredictable clutch operation bands $\omega \in<0.96,1>$ and $\omega \in\langle 1.478,1.565\rangle$. However, individual $2 \mathrm{~T}$-periodic solutions exist in the ranges of the control parameter $\omega \in\langle 0.689,0.704\rangle$ and $\omega \in<0.909,0.923\rangle$. Single $4 \mathrm{~T}$-periodic solutions can be found in intervals $\omega \in<1.258$, $1.287\rangle, \omega \in<1.69,1.707>$ and $\omega \in<1.965,2\rangle$. However, chaotic solutions take place in these ranges.

The mentioned areas of variation are given in a general way, and a detailed analysis of the DS diagram (Fig. 4a) indicates the sporadic presence of several coexisting solutions. Moreover, in the band $\omega<0.67$, there are occasional 1 T-periodic multiple solutions located in the vicinity of the frequency $\omega \approx 0.38, \omega \approx 0.5, \omega \approx 0.65$.

A comparison of the DS diagram and the one-dimensional distribution of the largest Lyapunov exponent (Fig. 4b) indicates significant differences in the variability of the control 
parameter $\omega \in<1.565,1.65\rangle$. In this range, $\lambda<0$ suggests that there are no indications of the presence of chaotic movement that actually exists. The difference must be explained with the criteria assuming the procedure for identification of the largest Lyapunov exponent. While drawing the distribution of the largest Lyapunov exponent, we accepted the constant value of the initial condition $u(0)=0, \dot{u}(0)=0$. However, the periodic solutions $P S$ value is calculated taking into account many different initial conditions. For this reason, the one-dimensional distribution of the largest Lyapunov exponent $\lambda$ in this range shows the presence of only a predictable solution. Apart from this difference, both diagrams show a significant compatibility and thus indicate the existence of three basic bands in which chaotic clutch movement takes place.

The first band (I zone) is in the range of variability $\omega \in<0.795,0.898>$, with a narrow periodic solution zone inside it. The widest area of irregular solutions (II zone) is within the frequency range from 1.035 to 1.226 . And the third zone includes a set of frequencies from 1.57 to 1.675 . Based on the periodicity of solutions PS (Fig. 4a), the values of a dimensionless frequency excitation were selected to obtain only a chaotic solution. Exemplary geometrical structures of attractors, representing permanent chaos, are shown graphically in diagrams (Fig. 5).

Graphical images of Poincaré cross sections were obtained based on 21,000 points to show the complexity of the chaotic attractor structures. For the qualitative evaluation of the points of intersection of the phase trajectory with the control plane, numerical tools are used to calculate the fractal dimension. In our research, we applied the correlation dimension, because it is more reliable in relation to the box size [36]. The analysis of Poincaré cross sections and $D_{C}$ correlation dimensions (Fig. 5) indicates that chaotic attractors assume the most complex structure in the third zone of unpredictable solutions. It is worth noting that in every zone, the chaotic attractor takes on a different geometrical form. Much more information about the geometrical structures of chaotic attractors is obtained by analysing the density of the distribution points of the trajectory with the control plane. The areas of the phase plane in which the cross-sectional points accumulate are located in places where the attractor is curved. Based on the numerical experiments, it can be concluded that with the increase in the dimensionless excitation frequency $\omega$, the geometrical structure of the chaotic attractors is more complex. This is confirmed by the value of the $D_{C}$ correlation dimension and the probability density function (PDF). As the dimensionless excitation frequency increases, the value of the correlation dimension also increases and the value of PDF decreases.

\subsection{Identification of multiple solutions}

In this section, examples of multiple periodic solutions of the nonlinear tyre clutch model are illustrated graphically using the corresponding basins of attraction (Fig. 6). The particular solutions are characterized by the period in the response expressed as the multiplicity of the excitation period. Similar coexistence can be obtained from multistable systems; however, in the case period one solutions are multiplied naturally [30, 31]. In the case when the system is affected by external excitation with a frequency of $\omega=0.677$, there are three coexisting solutions, one $1 \mathrm{~T}$-periodic and two $2 \mathrm{~T}$-periodic ones. If the frequency value $\omega$ increases to 0.772 , then three solutions are possible, one of which is $1 \mathrm{~T}$-periodic, the other $3 \mathrm{~T}$-periodic and the third $4 \mathrm{~T}$-periodic. However, above the frequency of $\omega>1$, stable solutions with periodicity of $8 \mathrm{~T}$ and $9 \mathrm{~T}$ appear.

The basins of attraction are usually crossed out with regard to singular points and attractors. To enable the mapping of the phase trajectory, it is enough to know the phase coordinates of one fixed point that corresponds to the given solution. In the case of harmonic responses, the fixed points do not change after passing iteratively from $\tau=n 2 \pi$ to $\tau=(n+1) 2 \pi$, whereas in relation to periodic responses, the phase stream crosses the Poincaré control plane after passing the $i$-time mapping. At the same time, the parameter $i$ represents the periodicity of the solution. From a theoretical point of view, these points can be interpreted as a special case of initial conditions. Assuming them, one can directly observe the phase trajectory, bypassing the time of transient motion. This property is characterized only by fixed points, in contrast to the others located on the phase trajectory.

The identification of basins of attraction is a time-consuming numerical procedure (Fig. 6). Nevertheless, having information about the phase coordinates of at least one determined point that uniquely defines the attractor of a given solution, it is possible to determine a set of initial conditions. In general terms, the procedure we apply consists of comparing the determined points of individual solutions with those calculated numerically on the basis of initial conditions. With a view to obtaining a satisfactory resolution, the range of phase coordinates variability was divided into 500 equal sections. As a result of such a procedure, 250,000 initial conditions were tested, based on which the basins of attraction corresponding to individual solutions were drawn. The colours we assigned to each set of initial conditions were set so that the final result of the numerical calculations was unambiguous for interpretation. For this reason, the existing $1 \mathrm{~T}$-periodic solutions have different colours 

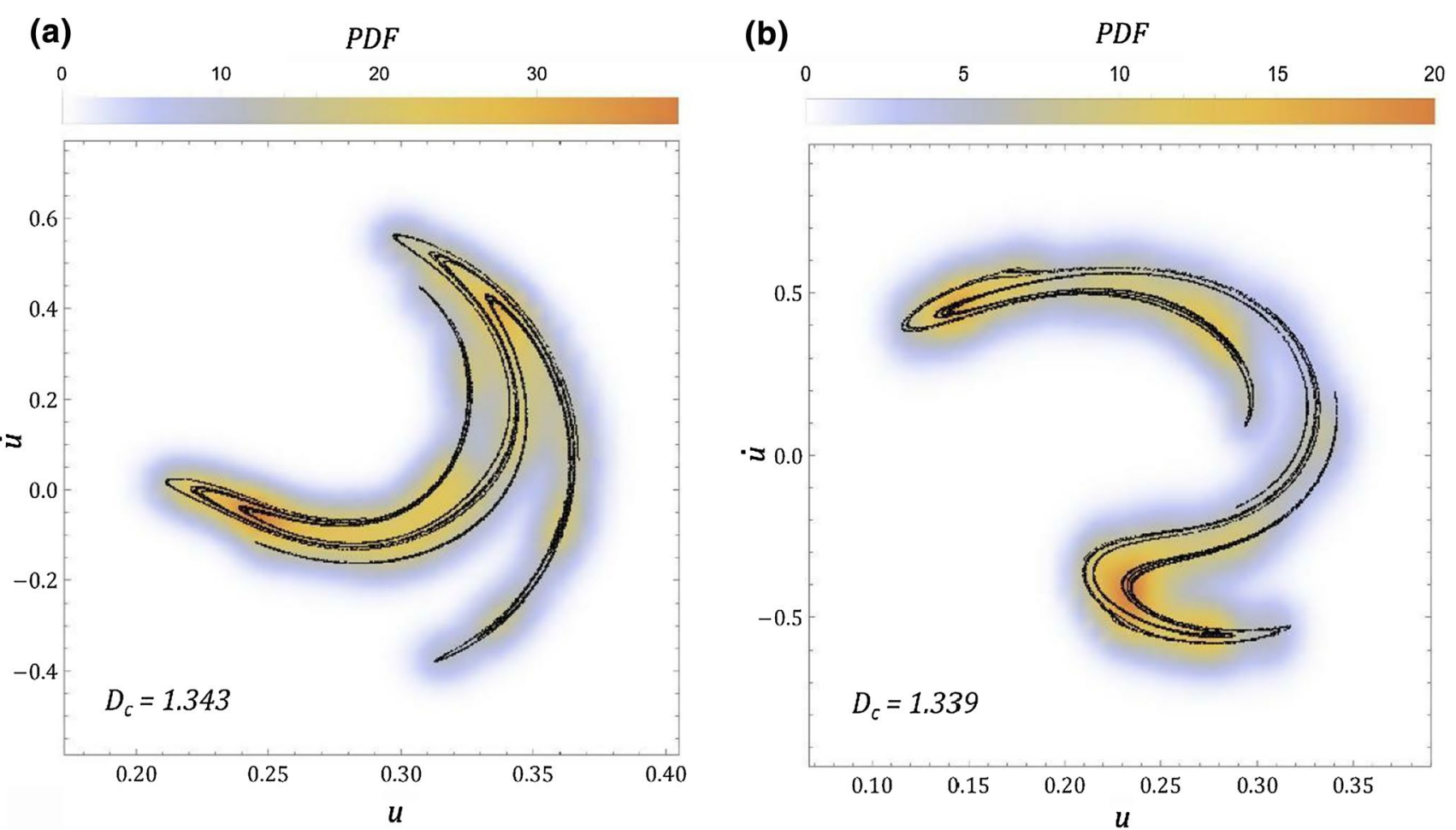

(c) PDF
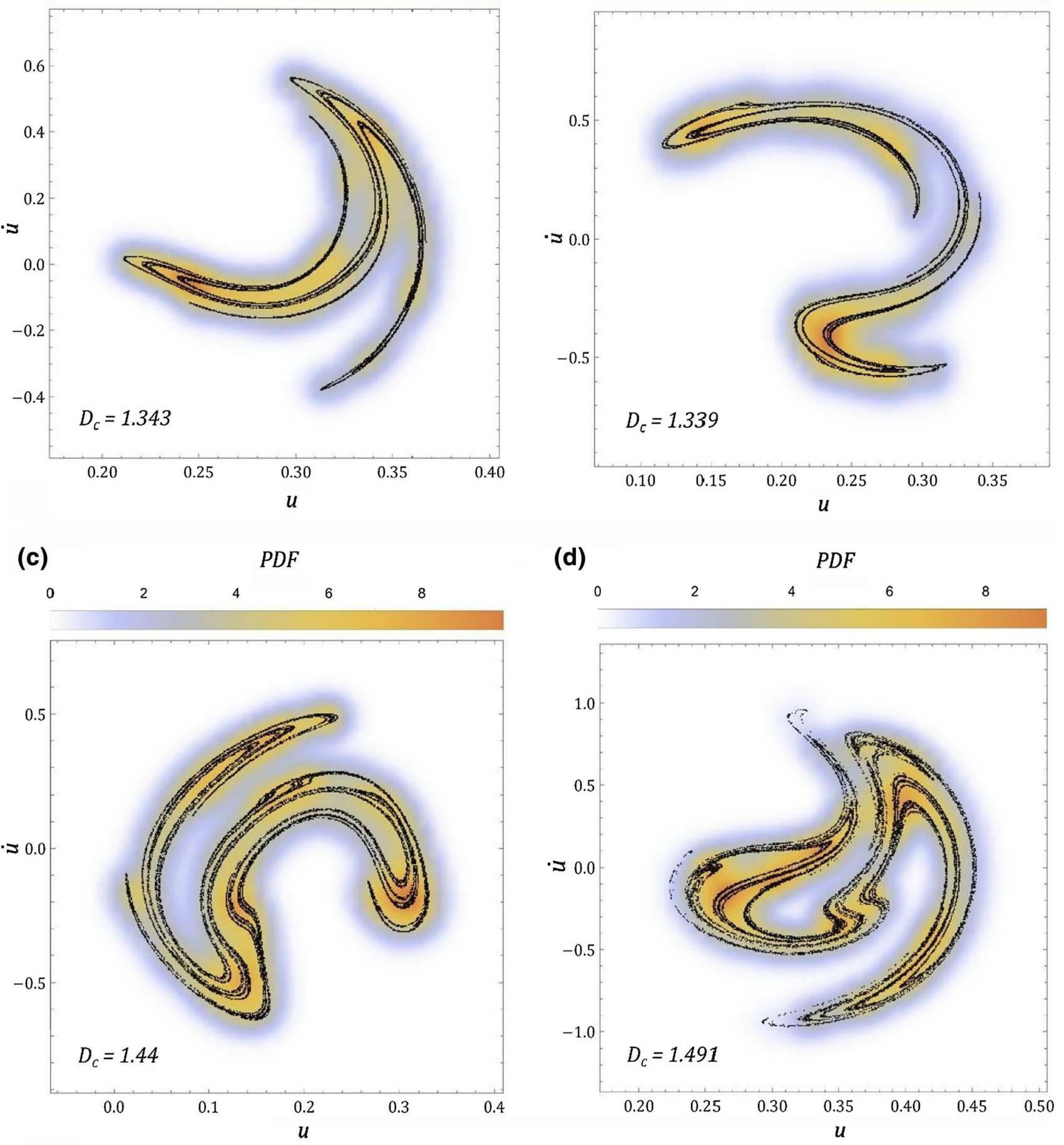

Fig. 5 Exemplary geometrical structures of chaotic attractors of the coupling model: a $\omega=0.835$, b $\omega=0.886, \mathbf{c} \omega=1.124$, d $\omega=1.657$, assuming initial conditions $(u(0)=0, \dot{u}(0)=0)$, where $D_{C}$ means the correlation dimension 

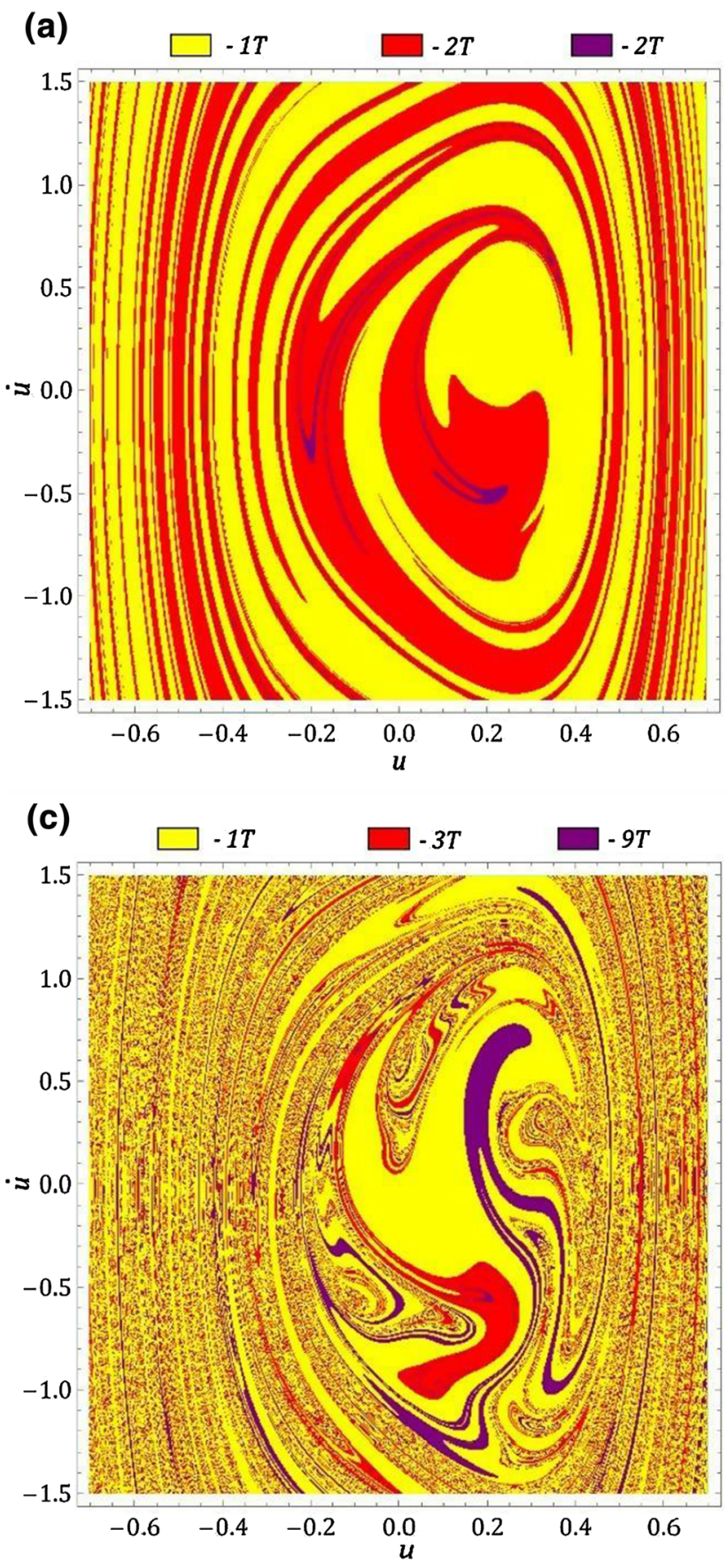

Fig. 6 Basins of attraction for multiple nonlinear clutch solutions: a $\omega=0.677, \mathbf{b} \omega=0.772$, c $\omega=1.42, \mathbf{d} \omega=1.8$. Note the typical Melnikov mixing of two colours (basins) in $\mathbf{b}, \mathbf{c}$ and $\mathbf{d}$. Wada features of

of $-0.5<u<0.5$. In this range, there are phase trajectories of the tested periodic solutions of the nonlinear coupling model.

At the lowest forced frequency we analysed $\omega=0.677$ (Fig. 6a), 1 T-periodic solutions (yellow colour) and the first 2 T-periodic solution (red colour) dominate. The second

assigned, depending on the frequency of the external load acting on the clutch.

Irrespective of the value of the external load frequency which affects the coupling, the basins of attraction in the variation intervals $u<-0.5$ and $u>0.5$ undergo a strong extension. Their clear shapes can be observed in the range
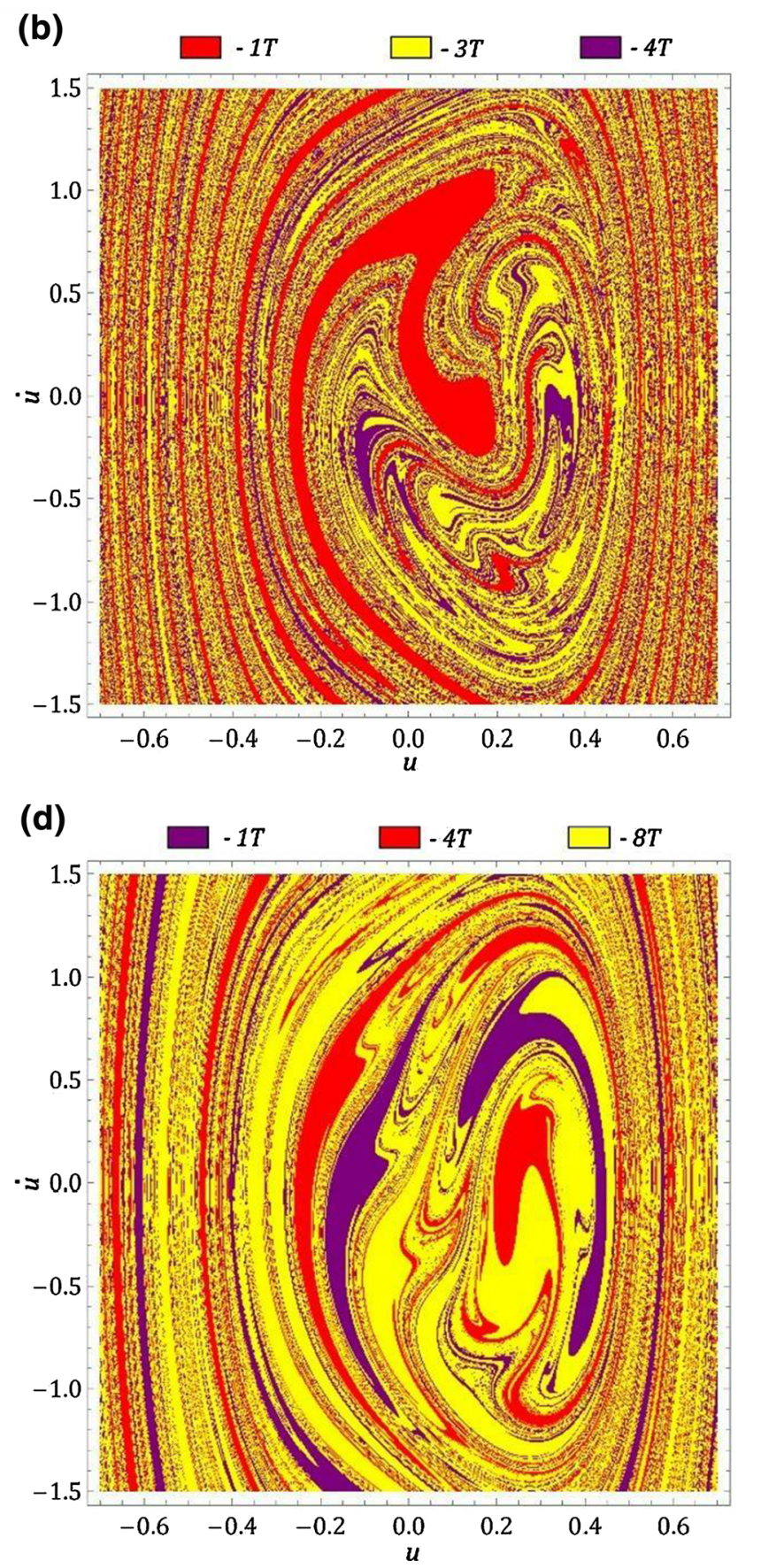

three colours mixing is visible in the same figures along the red-coloured basin edge 
2 T-periodic solution (violet colour) plays a secondary role. It is also worth noting that the set of initial conditions, corresponding to the second $2 \mathrm{~T}$-periodic solution (violet colour), is located inside the first set of $2 \mathrm{~T}$-periodic solutions (red colour). While using the terms "dominate" and "play a secondary role," we mean the size of the areas occupied by particular solutions and should be interpreted in this way. Relating them to the dynamics of the system is not justified, because the forces induced in the elastic coupling connector are primarily dependent on its current operating characteristics.

With respect to the other graphs (Fig. 6), the basins of attraction (Fig. 6b) corresponding to the 3 T-periodic (yellow) and the $4 \mathrm{~T}$-periodic (purple) solutions create small local concentrations. Any attempt to directly compare the size of individual sets, without detailed numerical calculations, will be burdened with a large error. It is possible to state, however, that the set of initial conditions reflecting the $1 \mathrm{~T}$-periodic solutions (red colour) basically creates a uniform coherent region, which in time extends extensively. If there is an external load acting on the coupling with frequency $\omega=1.42$ (Fig. 6c), then the largest area of initial conditions is assigned to the $1 \mathrm{~T}$-periodic solution (yellow colour).

The situation is completely different when the load is driven by the frequency $\omega=1.8$ (Fig. 6d). In this case, the first set of initial conditions corresponding to the 8 T-periodic solution (yellow colour) dominates. Basins of attraction with $1 \mathrm{~T}$-periodic (purple) and $4 \mathrm{~T}$-periodic (red) solutions are definitely smaller. The results presented in Fig. 6b-d clearly illustrate the fractal boundaries of three basins. These basins have the property of Wada [37-39]. The border point of one of the basins also belongs to the border of three different basins of attraction (along the red-coloured basin edge). In such cases, the dynamics of the considered system becomes even more complex.

\subsection{Non-permanent chaotic and periodic solutions}

In addition to the possibility of identifying the number of coexisting solutions and stable periodic orbits, diagrams of periodic solutions DPS also indicate the places in which the dynamics of the system changes over time. Thus, we are referring to the transition of a chaotic solution into a periodic solution. To clearly establish the presence of unstable chaotic behaviour, it is necessary to carry out additional studies of all coexisting solutions characterized by high periodicity. A detailed analysis of coexisting solutions was carried out with reference to three values of the control parameter $\omega$.

If the external coupling force with the frequency $\omega=0.792316$ affects the clutch model, which is located in close proximity to the I zone of permanent chaotic solutions, then three different system responses are possible.
Of those that are distinguished by two permanent $1 \mathrm{~T}$-periodic solutions $(u=0.3464, \dot{u}=0.0049)$ and $3 \mathrm{~T}$-periodic ( $u=0.2507, \dot{u}=0.1071$ ). At the same time, they will not be the subject of our detailed inquiries, because we focus our attention on non-permanent solutions.

The third 25 T-periodic solution reflects the phenomenon of transient chaos ( $u=0.2886, \dot{u}=-0.082)$. However, as time passes, it is attracted to the $1 \mathrm{~T}$-periodic orbit (Fig. 7a). The correlation dimension of the attractor mapping the transition of the chaotic solution to the periodic solution takes the value after the rounding of $D_{C}=0.3352$. The frequency with which the system was forced is located just before the first zone of irregular solutions, and the geometrical structure of the Poincaré cross section is similar to the "poorer" version of the graph (Fig. 5a). The Poincaré cross section, whose geometrical structure is represented in blue, represents a permanent chaotic solution.

Direct comparison of the two sections indicates how the chaotic attractor will evolve as the value of the control parameter $\omega$ increases. On this basis, it can be concluded that due to the increase in the value of $\omega$ the chaotic solution begins to play the dominant role. In contrast, the chaotic attractor is subject to stretching.

The formal basis for assessing the evolution of harmonic components, over time, is a scalogram (Fig. 7b), for which a Gabor wavelet was used. Based on it, the dominant frequencies and the highest activity bands were determined. In addition, the time $\tau \approx 2750$ of transient chaos is estimated approximately. However, this approximation is sufficient. Precise evaluation of $\tau$, based on a scalogram, is burdened with an error resulting from the procedure of generating a two-dimensional spectrum. In general terms, the identification of STFT spectra boils down to the multiple calculation of the classic Fourier's spectrum, based on a sequence of samples determined by the width of the moving time window. For this reason, in the vicinity of the point, the transition of the chaotic solution to the periodic solution is observed as a "blur" of the spectrum, which makes it difficult to determine the time $\tau$.

A much more accurate value can be determined based on the stroboscopic image of the Poincare cross section, which is observed in coordinates $(\tau, u)$ or $(\tau, \dot{u})$. In the analysed signal, the excitation frequency $\omega_{1}=0.792316$ and the harmonic component $\omega_{2} \approx 0.12$ play an essential role. A cause-and-effect relationship $\omega_{1}=6.5 \omega_{2}$ exists between the two frequencies. In the amplitude-frequency spectrum calculated on the basis of the signal containing the transient chaos phenomenon (dark blue colour of the spectrum), subharmonics and additional frequencies are excited, between which the cause-and-effect relationships appear on the graph (Fig. 7c). Over time, the transient chaos is "extinguished" and transformed into a $1 \mathrm{~T}$-periodic solution (red spectrum). Comparison of both Fourier's spectra shows that due to 
(a)

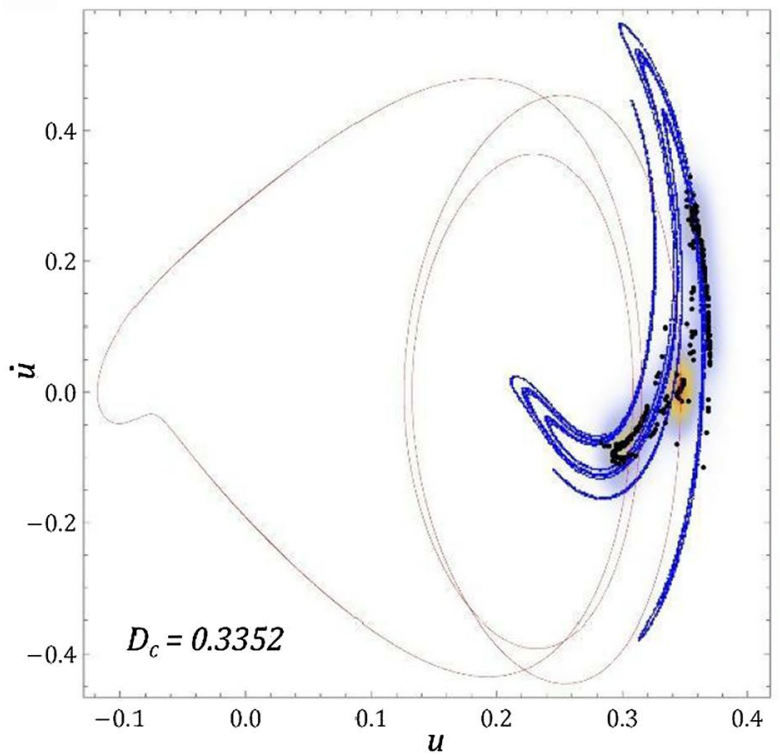

(c)
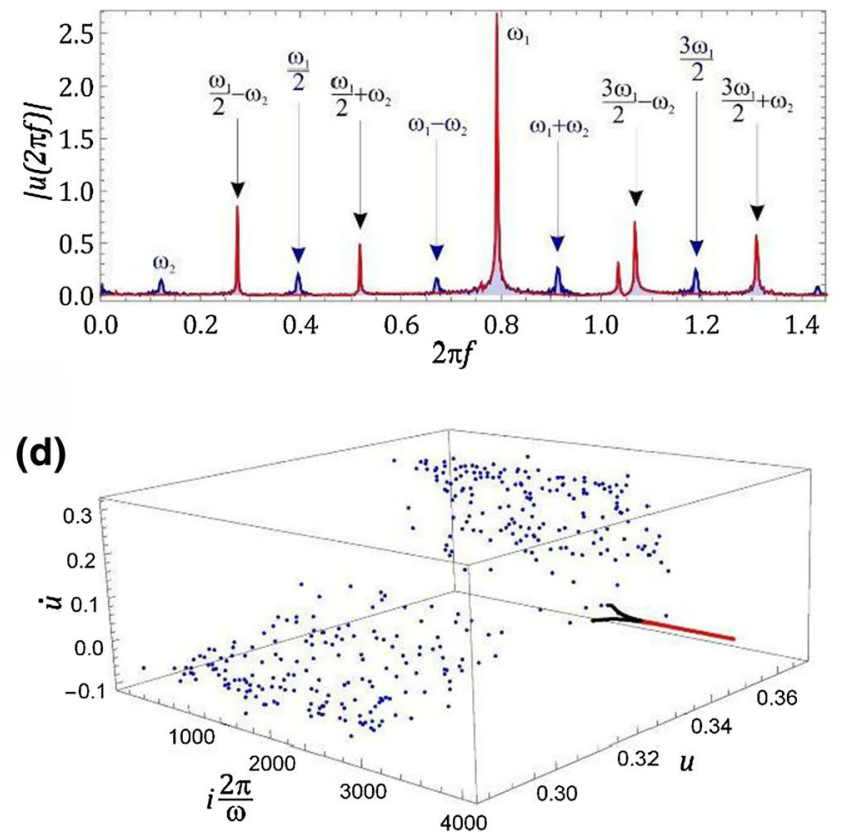

(b)

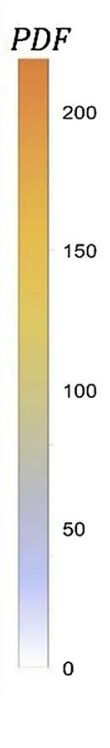

(e)

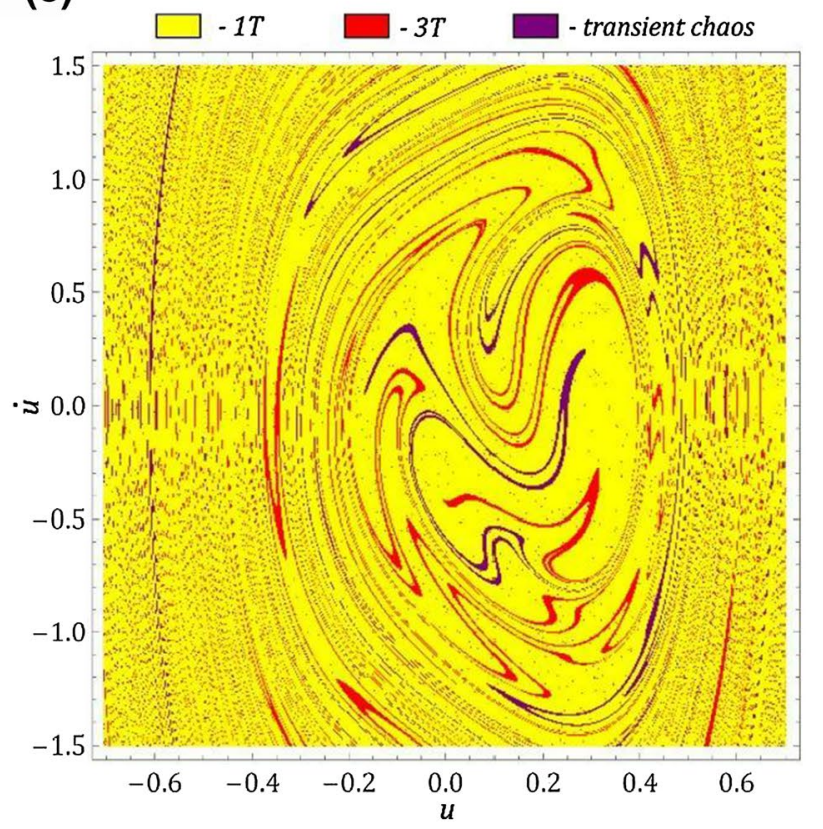

Fig. 7 Results of computer simulations for $\omega=0.792316$ : a Poincaré cross section and 1 T-periodic phase trajectory, b timescale scalogram, c amplitude-frequency spectrum, $\mathbf{d}$ stroboscopic Poincaré cross section, e basins of attraction

changes in the dynamics of the system some harmonic components are suppressed. On the graph (Fig. 7d), the points of intersection of the phase trajectory with the control plane are depicted in the form of a stroboscopic diagram. In fact, this is a graphic image of the Poincaré section (Fig. 7a), but is presented in the form of a three-dimensional stroboscopic chart. Its detailed analysis indicates that the chaotic response (blue points) in $1 \mathrm{~T}$-periodic (red points) takes place through a 2 T-periodic solution (black points). However, the time of recorded 2 T-periodic vibrations of the coupling is relatively short and amounts to approx. 60 periods of excitation.

This character of the dynamics of the tested model can be observed only when the Poincaré cross section is presented in the form of a stroboscopic plot. It is also worth noting that this information is not provided by the research tool we used, which is short-term frequency analysis (Fig. 7b). With regard to three coexisting solutions, the basins of attraction corresponding to a given solution are plotted in Fig. 7e. 
The identified basins are basically composed of uniform, consistent areas, and the largest of them corresponds to a $1 \mathrm{~T}$-periodic solution (yellow). At the same time, randomly scattered initial conditions corresponding to 3 T-periodic vibrations (red colour) and transient chaos (purple colour) are located inside it.

When the external load acts on the clutch with a frequency of $\omega=1.2344$, the value of which lies just after the II zone of the permanent chaotic solutions, two

(a)

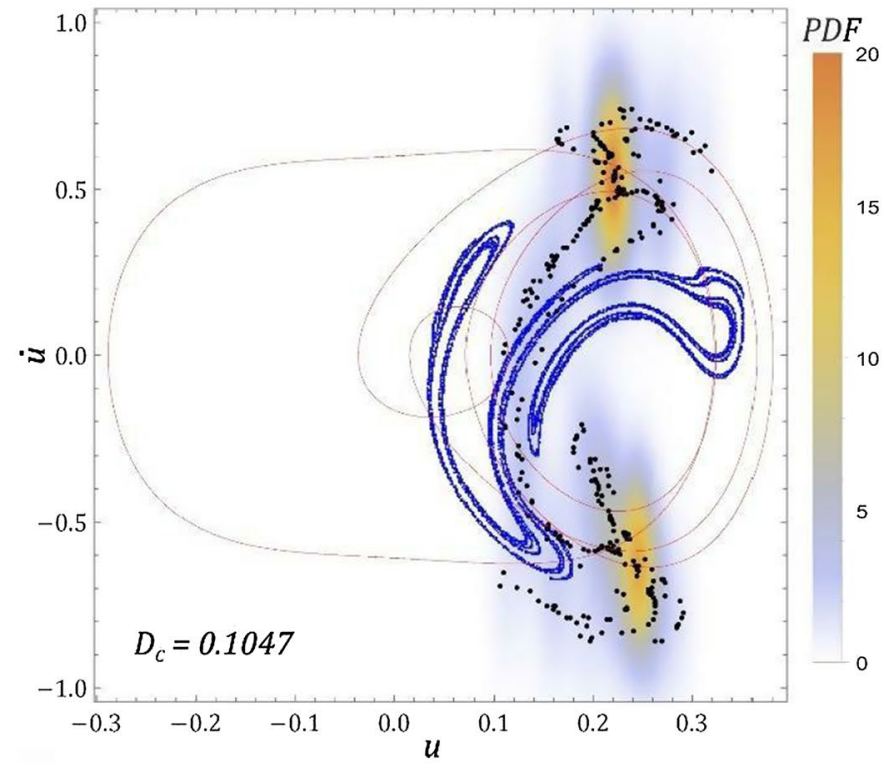

(c)
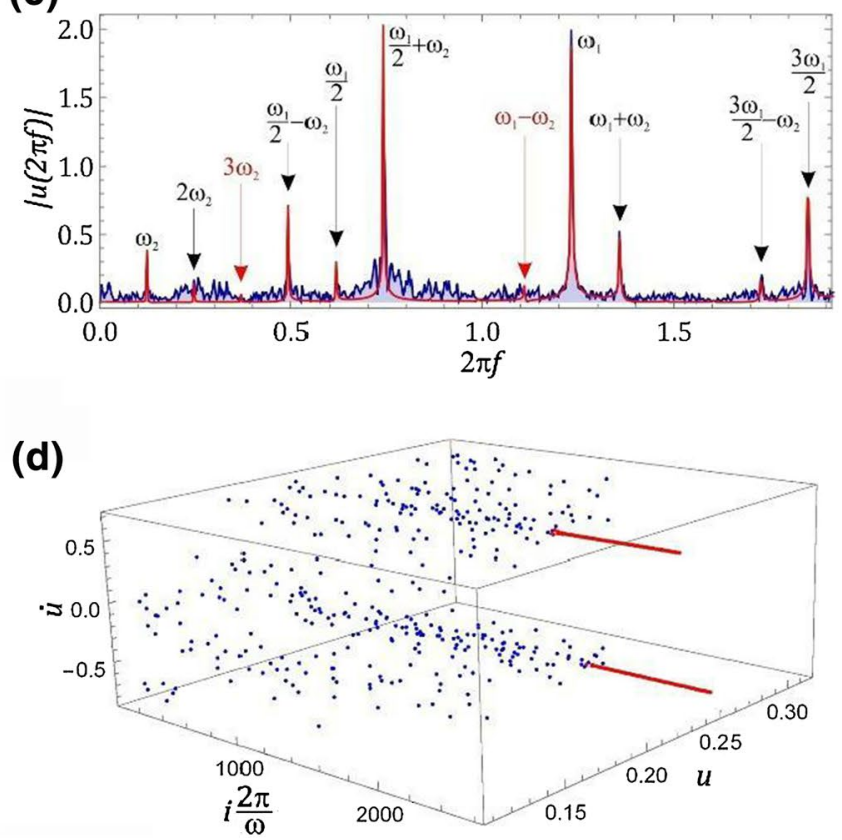

stable 2 T-periodic solutions coexist. The first is defined by a fixed point $(u=0.35, \dot{u}=-0.2051)$ and the second $(u=0.2215, \dot{u}=0.5401)$. The third solution ( $u=0.1246, \dot{u}=-0.2745$ ) reflects the phenomenon of transient chaos, which over time is attracted to the second $2 \mathrm{~T}$-periodic solution. The results of computer simulations are presented in Fig. 8.

The geometrical structure of the unstable chaotic attractor (Fig. 8a) was compared with the graphic image of the (b)

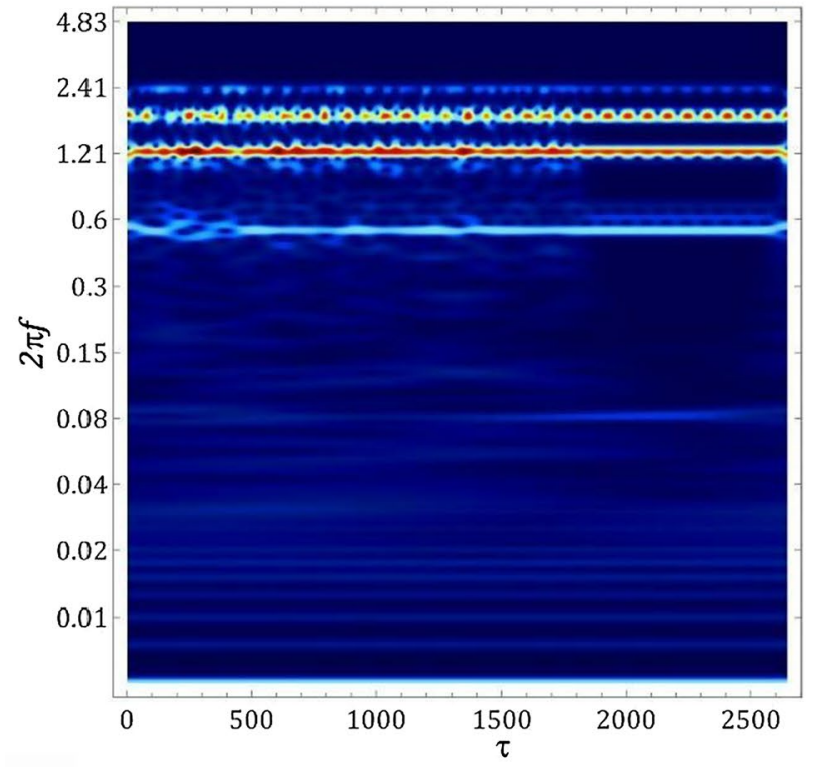

(e)

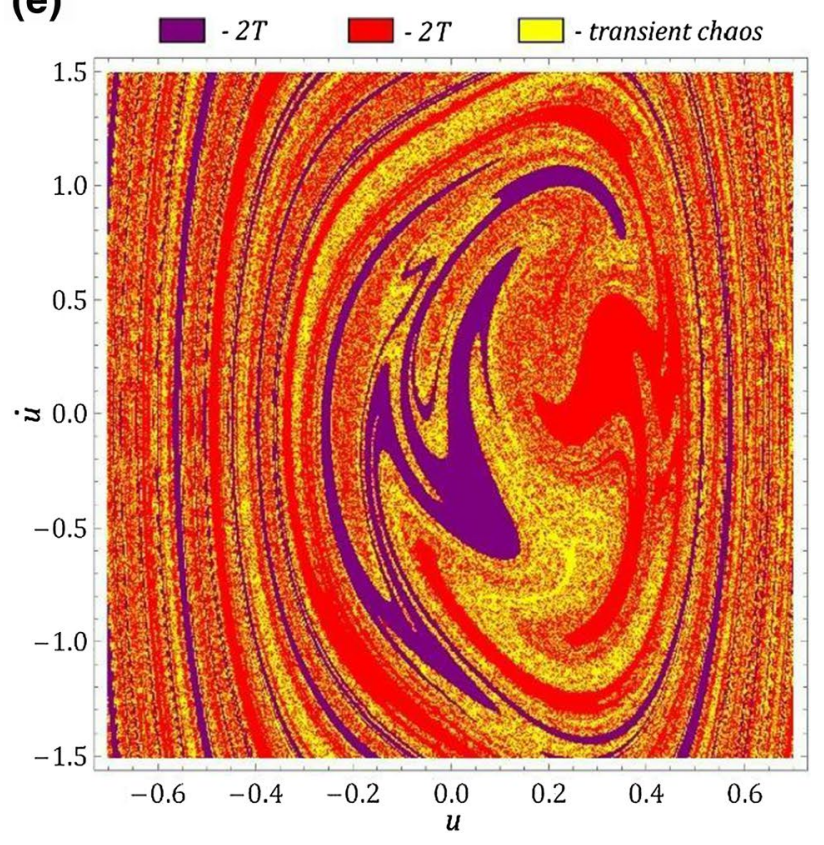

Fig. 8 Results of computer simulations for $\omega=1.2344$ : a Poincaré cross section and 1 T-periodic phase trajectory, b timescale scalogram, c amplitude-frequency spectrum, $\mathbf{d}$ stroboscopic Poincaré cross section, e basins of attraction 
Poincaré cross section, which maps the permanent chaos at the end of the second zone. Their direct reference indicates a slight similarity, and any attempt to prove that it is the same attractor subjected to metamorphosis may be fraught with errors. The correlation dimension of the transient chaotic attractor assumes a value approximately equal to $D_{C} \approx 0.1$ (Fig. 8a), and this value is much smaller in relation to the previously analysed example (Fig. 7a). At the same time, the location and the number of points in which the phase trajectory crosses the Poincare control plane determine the value of the correlation dimension.

Based on the scalogram (Fig. 8b), the transition time $\tau$ $\approx 1800$ of the chaotic solution to the periodic solution with the period equal to 2 was identified. In relation to the previously analysed load case, the frequency-amplitude spectrum (Fig. 8c) is characterized by continuous decay. Nevertheless, subharmonics of excitation $\omega_{1}$ are forced. The dominant harmonic components have been described through the cause-and-effect relations between the frequencies $\omega_{1}$ and $\omega_{2}$. The dependencies presented in black appear both in the part of the signal that corresponds to the unstable chaos (the spectrum in navy blue) and in the stable second $2 \mathrm{~T}$-periodic solution.

As time goes by, the frequency-amplitude spectrum is transformed (red spectrum), and the corresponding towering frequencies in it are marked in red. It is worth mentioning that between the frequency $\omega_{1}$ and $\omega_{2}$, the following cause-and-effect relationship is present: $\omega_{1}=10 \omega_{2}$. The stroboscopic representation of the unstable chaotic solution (Fig. 8d) unambiguously indicates to which fixed points the intersections of the phase stream with the control plane are attracted. In order to ensure the readability of basins of attraction, the convention so far used has been changed (Fig. 8e). Based on the analyses carried out, it was found that only the basins of attraction of the first 2 T-periodic solution creates a uniform coherent domain (purple). In the case of the second $2 \mathrm{~T}$-periodic solution (red colour), there is also a uniform area. Nevertheless, the remainder of the considered phase space includes the initial conditions, which are mixed with the conditions corresponding to the transient chaos (yellow colour).

Assuming that the clutch is affected by an external drive torque with a frequency of $\omega=1.9013$, which is located at the end of the tested variation range of the control parameter, three identical 2 T-periodic solutions coexist in the case study $(u=0.3978, \dot{u}=0.1911), 4 \mathrm{~T}$-periodic ( $u=0.1757, \dot{u}=-0.0992)$ and $4 \mathrm{~T}$-periodic solutions attracted to the attractor, corresponding to transient chaos ( $u=0.1523, \dot{u}=0.1186$ ). Despite the considerable distance from the main zone III of the chaotic movement, the graphic image of the intersection points of the phase stream with the control plane with its geometrical shape slightly resembles the "poorer and stretched" section of the Poincare section
(Fig. 5d). Bearing in mind the devotion of this modest similarity, it was depicted on the background of a chaotic attractor (points in blue) dominating in the third zone of irregular solutions (Fig. 9a). This is a relatively small similarity of attractors, which is explained by the relatively large distance from the third zone of chaotic movement. On the basis of the short-term frequency analysis, the results of which were presented in the form of a scalogram (Fig. 9b), it was found that the transition time of the chaotic to periodic solution is approximately $\tau \approx 1250$.

Based on the scalogram, it is possible to distinguish only the basic harmonic components responsible for the nature of the solution. For this reason, the amplitude-frequency characteristics were calculated (Fig. 9c), the signals corresponding to the transient chaos (the dark blue colour of the spectrum) and the $4 \mathrm{~T}$-periodic solution (the red spectrum colour). Based on the identified frequencies, it was found that in the spectrum beyond the frequency of external load $\omega_{1}$ acting on the coupling and $\omega_{2}$, there are additionally sub- and interharmonics of frequency $\omega_{1}$. The black colour indicates the harmonic components occurring in the both transient chaos and the periodic signal. In addition, in the amplitude-frequency spectra there are components that are a combination of the fundamental frequency $\omega_{1}$ and $\omega_{2}$. For the sake of clarity, they have not been marked in the spectra. With the transition of the chaotic to the periodic solution, the combinations of interharmonics frequencies $\omega_{1}$ and $\omega_{2}$ are suppressed. Similarly to Fig. 8, between the frequencies $\omega_{1}$ and $\omega_{2}$, there is still a cause-and-effect relationship $\omega_{1}=10 \omega_{2}$.

The stroboscopic Poincaré cross-sectional visualization indicates that transient chaos transforms into a $4 \mathrm{~T}$-periodic solution directly, bypassing the bifurcation transition (Fig. 9d). The analysis of the basins of attraction clearly indicates that in the case of a clutch load with a frequency of $\omega=1.9013$, the $4 \mathrm{~T}$ and $2 \mathrm{~T}$-periodic solutions dominate (Fig. 9e). The initial conditions corresponding to the unstable chaos do not form a uniform compact set, but are moved with the conditions of the $4 \mathrm{~T}$-periodic solution. The drawn basin of attraction resembles the geometrical structure of the chaotic attractor. Similar behaviour, in some areas of the analysed phase space, takes place in relation to the basin of attraction of the $2 \mathrm{~T}$-periodic solution.

\section{Conclusions}

The phenomenon of transient chaos occurs most frequently in the ranges of variability of the control parameter preceding and ending the main zones of the presence of permanent chaotic solutions. The results of the model research presented in this paper were limited to discussing the example of the coexistence of two or three stable periodic and 
(a)

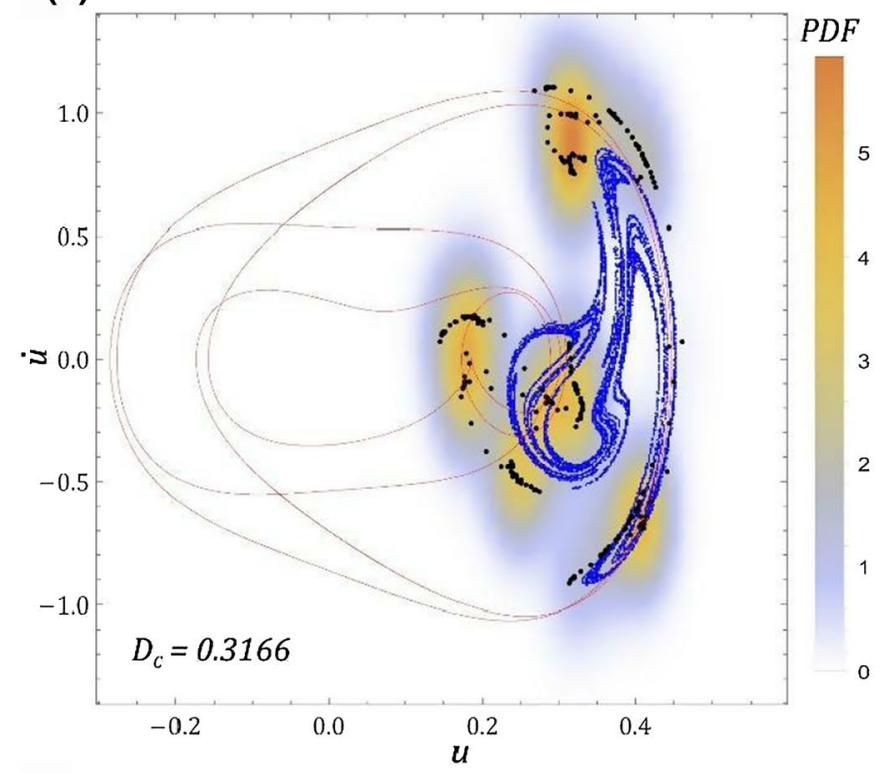

(c)

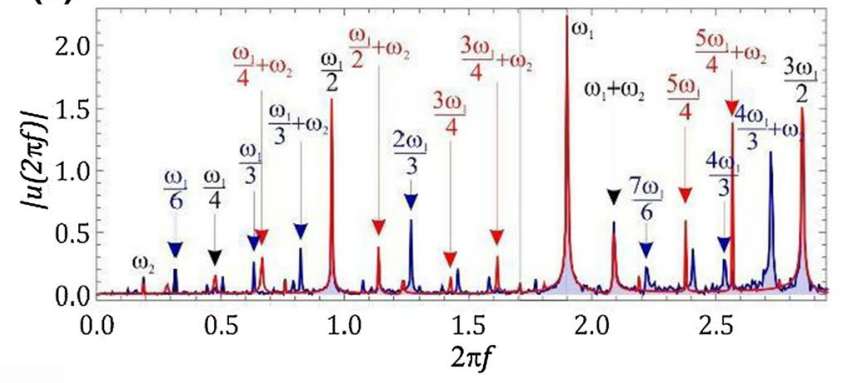

(d)

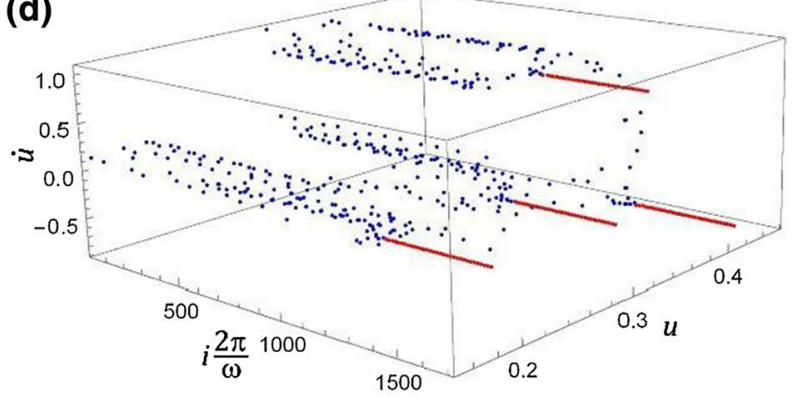

(b)

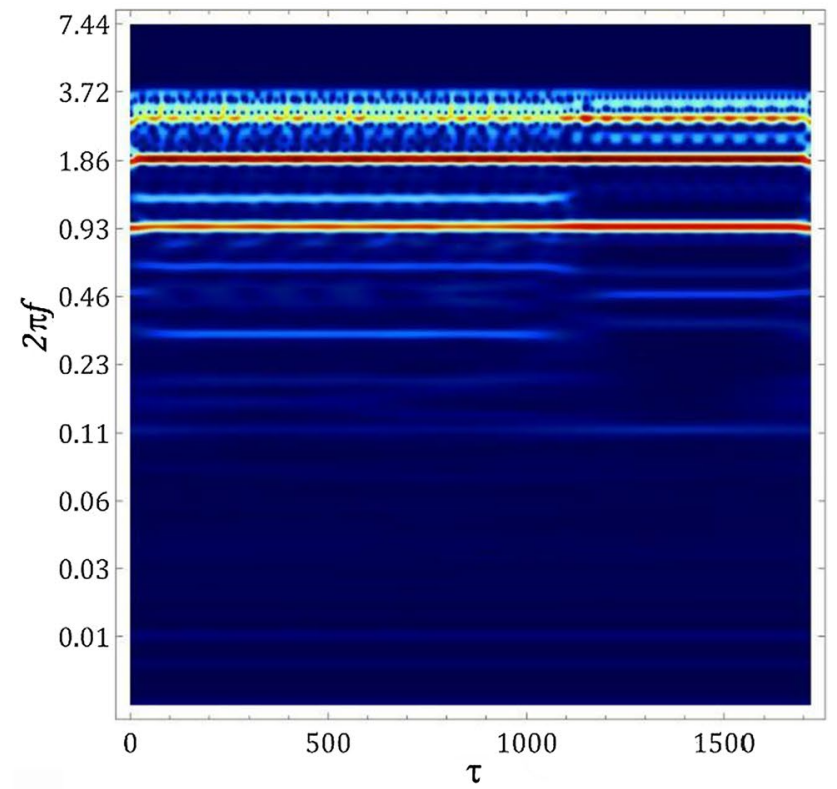

(e)

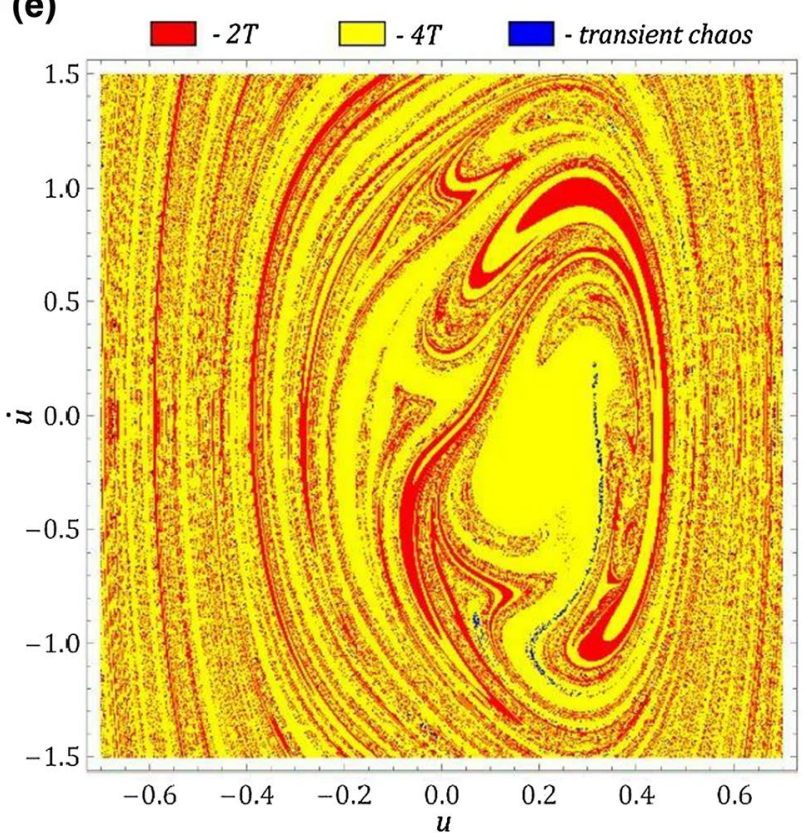

Fig. 9 Results of computer simulations for $\omega=1.9013$ : a Poincaré cross section and 1 T-periodic phase trajectory, b timescale scalogram, c amplitude-frequency spectrum, $\mathbf{d}$ stroboscopic Poincaré cross section, e basins of attraction

transient solutions that mapped the phenomenon of transient chaos. It was limited to presenting several cases with three solutions due to the readability of the basins of attraction. This was also done due to the fact that in some situations the time of non-permanent chaos is relatively short, and it is difficult to distinguish a chaotic solution from the unchanged working time of a clutch. To sum up, based on the conducted model tests of nonlinear dynamics of the flexible coupling, it is possible to formulate the following conclusions:
- Diagrams of solutions (periodicity and the number of solutions) DS provide new qualitative information about the dynamics of the system.

- Regarding the one-dimensional distribution of the largest Lyapunov exponent, DS diagram deal with many initial conditions, as a result of which the identification of chaotic motion zones is more accurate.

- Based on DS diagrams, it is possible to identify solutions reflecting the phenomenon of transient chaos. 
- It was found that this phenomenon occurs most often when the DS diagram (shaded by a light blue colour in Fig. 4a) indicates a small number of possible solutions and informs about the existence of solutions of low and high periodicity. Such cases require a detailed analysis, due to the coexistence of many periodic solutions and permanent chaos.

- In the graphic images of the basins of attraction, the areas corresponding to the transient and periodic chaos are blurred.

From an operational point of view, and in particular the nature of the operation of a drive system, the phenomenon of transient chaos can be significant or can be neglected. In the case of drive systems of handling machines that perform intermittent motion (i.e. cranes), neglecting transient chaos is impossible because in such machines this phenomenon plays a key role. However, in relation to belt conveyors and devices working in a continuous motion, they can be neglected, because as time goes on, the chaotic solutions are "extinguished" and transform into predictable periodic vibrations. It is worth considering that the value of the correlation dimension is influenced by the duration of the transient chaos and the frequency of excitation of the external load.

\section{Declarations}

Conflict of interest The authors declare that they have no conflict of interest.

Open Access This article is licensed under a Creative Commons Attribution 4.0 International License, which permits use, sharing, adaptation, distribution and reproduction in any medium or format, as long as you give appropriate credit to the original author(s) and the source, provide a link to the Creative Commons licence, and indicate if changes were made. The images or other third party material in this article are included in the article's Creative Commons licence, unless indicated otherwise in a credit line to the material. If material is not included in the article's Creative Commons licence and your intended use is not permitted by statutory regulation or exceeds the permitted use, you will need to obtain permission directly from the copyright holder. To view a copy of this licence, visit http://creativecommons.org/licenses/by/4.0/.

\section{References}

1. Serkies P (2017) Comparison of the control methods of electrical drives with an elastic coupling allowing to limit the torsional torque amplitude. Eksploat i Niezawodn Maint Reliab 19:203210. https://doi.org/10.17531/ein.2017.2.7.

2. Margielewicz J, Opasiak T, Gąska D, Litak G (2019) Study of flexible couplings nonlinear dynamics using bond graphs. Forsch Im Ingenieurwes. https://doi.org/10.1007/s10010-019-00317-w

3. Zhao Z, He L, Yang Y, Wu C, Li X, Hedrick JK (2016) Estimation of torque transmitted by clutch during shifting process for dry dual clutch transmission. Mech Syst Signal Process 75:413-433. https://doi.org/10.1016/j.ymssp.2015.12.027

4. Zhou J, Sun W, Yuan L. Nonlinear Vibroimpact Characteristics of a Planetary Gear Transmission System. Shock Vib 2016;2016. https://doi.org/10.1155/2016/4304525.

5. Lorenz EN (1963) Deterministic nonperiodic flow. J Atmos Sci 20:130-141

6. Khomeriki G (2016) Parametric resonance induced chaos in magnetic damped driven pendulum. Phys Lett Sect A Gen At Solid State Phys 380:2382-2385. https://doi.org/10.1016/j.physleta. 2016.05.049

7. Awrejcewicz J, Krysko V Jr, Yakovleva V, Krysko VA (2017) Alternating chaos versus synchronized vibrations of interacting plate with beams. Int J Non Linear Mech 88:21-30. https://doi. org/10.1016/j.ijnonlinmec.2016.10.007

8. Borowiec M, Litak G, Friswell MI (2006) Nonlinear response of an oscillator with a magneto-rheological damper subjected to external forcing. Appl Mech Mater 5-6:277-284. https://doi.org/ 10.4028/www.scientific.net/AMM.5-6.277

9. Litak G, Borowiec M, Friswell MI, Szabelski K (2008) Chaotic vibration of a quarter-car model excited by the road surface profile. Commun Nonlinear Sci Numer Simul 13:1373-1383. https:// doi.org/10.1016/j.cnsns.2007.01.003

10. Armand Eyebe Fouda JS, Bodo B, Djeufa GMD, Sabat SL (2016) Commun Nonlinear Sci Numer Simulat Experimental chaos detection in the Duffing oscillator. Commun Nonlinear Sci Numer Simul 33:259-269. https://doi.org/10.1016/j.cnsns.2015.09.011.

11. Iqbal S, Zang X, Zhu Y, Zhao J (2014) Bifurcations and chaos in passive dynamic walking: a review. Rob Auton Syst 62:889-909. https://doi.org/10.1016/j.robot.2014.01.006

12. Rodríguez-Bermúdez G, García-Laencina PJ (2015) Analysis of EEG signals using nonlinear dynamics and chaos: a review. Appl Math Inf Sci 9:2309-2321. https://doi.org/10.12785/amis/090512.

13. Vlad S, Pascu P, Morariu N (2010) Chaos models in economics. J Comput 2:79-83

14. Stefanski A, Dabrowski A, Kapitaniak T (2005) Evaluation of the largest Lyapunov exponent in dynamical systems with time delay. Chaos Solitons Fractals 23:1651-1659. https://doi.org/10.1016/j. chaos.2004.06.051

15. Wolf A, Swift JB, Swinney HL, Vastano JA (1985) Determining Lyapunov exponents from a time series. Phys D Nonlinear Phenom 16:285-317. https://doi.org/10.1016/0167-2789(85)90011-9

16. Chang-Jian CW (2012) Bifurcation and chaos analysis of the porous squeeze film damper mounted gear-bearing system. Comput Math Appl 64:798-812. https://doi.org/10.1016/j.camwa. 2011.12.027

17. Margielewicz J, Gąska D, Litak G (2019) Modelling of the gear backlash. Nonlinear Dyn. https://doi.org/10.1007/ s11071-019-04973-z

18. Dudkowski D, Jafari S, Kapitaniak T, Kuznetsov N V., Leonov GA, Prasad A (2016) Hidden attractors in dynamical systems, vol. 637. Elsevier, Amsterdam. https://doi.org/10.1016/j.physrep.2016. 05.002 .

19. Margielewicz J, Gąska D, Litak G (2019) Evolution of the geometric structure of strange attractors of a quasi-zero stiffness vibration isolator. Chaos Solitons Fractals 118:47-57. https://doi.org/10. 1016/j.chaos.2018.11.012

20. Mohammed OD, Rantatalo M (2016) Dynamic response and time-frequency analysis for gear tooth crack detection. Mech Syst Signal Process 66-67:612-624. https://doi.org/10.1016/j.ymssp. 2015.05.015

21. Lonkwic P, Łygas K, Wolszczak P, Molski S, Litak G (2017) Braking deceleration variability of progressive safety gears using statistical and wavelet analyses. Measurement 110:90-97. https:// doi.org/10.1016/j.measurement.2017.06.005 
22. Figlus $\mathrm{T}$ (2019) A method for diagnosing gearboxes of means of transport using multi-stage filtering and entropy. Entropy, 21. https://doi.org/10.3390/e21050441.

23. Savi MA, Pereira-Pinto FHI, Viola FM, Souza de Paula A, Bernardini D, Litak G et al (2017) Using 0-1 test to diagnose chaos on shape memory alloy dynamical systems. Chaos, Solitons Fractals 103:307-324. https://doi.org/10.1016/j.chaos.2017.06.016.

24. Cattani M, Caldas IL, de Souza SL, Iarosz KC (2016) Deterministic Chaos theory: basic concepts. Rev Bras Ensino Física 39:1-13. https://doi.org/10.1590/1806-9126-rbef-2016-0185

25. Wright JA, Deane JHB, Bartuccelli M, Gentile G (2015) Basins of attraction in forced systems with time-varying dissipation. Commun Nonlinear Sci Numer Simul 29:72-87. https://doi.org/10. 1016/j.cnsns.2015.04.021

26. Zhu L, Raghu A, Lai Y (2001) Experimental observation of superpersistent Chaotic transients. Phys Rev Lett 86:4017-4020. https://doi.org/10.1103/PhysRevLett.86.4017

27. Woltering M, Markus M (2000) Riddled-like basins of transient Chaos. Phys Rev Lett 84:630-633. https://doi.org/10.1103/PhysR evLett.84.630

28. Sabarathinam S, Thamilmaran K (2015) Transient chaos in a globally coupled system of nearly conservative Hamiltonian Duffing oscillators. Chaos Solitons Fractals 73:129-140. https://doi.org/ 10.1016/j.chaos.2015.01.004

29. Souza de Paula A, Savi MA, Pereira-Pinto FHI (2006) Chaos and transient chaos in an experimental nonlinear pendulum. J Sound Vib 294:585-595. https://doi.org/10.1016/j.jsv.2005.11.015.

30. Lai Y-C, Tel T (2011) Transient Chaos: complex dynamics on finite-time scales. Springer, New York. https://doi.org/10.1007/ 978-1-4419-6987-3.

31. Santana L, da Silva RM, Albuquerque HA, Manchein C (2021) Transient dynamics and multistability in two electrically interacting FitzHugh-Nagumo neurons. Chaos 31:053107. https:// doi.org/10.1063/5.0044390

32. Melnikov VK (1963) On the stability of the center for time periodic perturbations. Trans Moscow Math Soc 12:1

33. Guckenheimer J, Holmes JP (1983) Nonlinear oscillations, dynamical systems and bifurcations of vectorfields. Springer, New York.

34. CAK Kwuimy, C Nataraj, G Litak, Melnikov's criteria, parametric control of chaos, and stationary chaos occurrence in systems with asymmetric potential subjected to multiscale type excitation, Chaos 21 (4), 043113, 2011

35. Grebogi C, Ott E, Yorke JA (1983) Crises, sudden changes in chaotic attractors, and transient chaos. Phys D Nonlinear Phenom 7:181-200

36. Tarnopolski M (2014) On the fractal dimension of the duffing attractor. Rom Reports Phys 66:907-917

37. Aguirre J, Vallejo JC, Sanjuan MAF (2001) Wada basins and chaotic invariant sets in the Henon-Heiles system. Phys Rev E 64:066208.

38. Litak G, Coccolo M, Friswell MI, Ali SF, Adhikari S, Lees AW, Bilgen O (2012) Nonlinear oscillations of an elastic inverted pendulum 2012 IEEE 4th International Conference on Nonlinear Science and Complexity (NSC), pp 113-116.

39. Syta A, Litak G, Lenci S, Scheffler M (2014) Chaotic vibrations of the duffing system with fractional damping, Chaos 24, 013107.

Publisher's Note Springer Nature remains neutral with regard to jurisdictional claims in published maps and institutional affiliations. 